\title{
PI 7-0 I. HIV-specific immune responses induced by a single dose of HIV DNA vaccine in Rhesus macaques
}

\author{
G Arrode-Brusés ${ }^{1}$, D Sheffer ${ }^{1}$, R Hegde ${ }^{1}$, Z Liu $^{1}$, F Villinger ${ }^{2}$, O Narayan ${ }^{1}$ and \\ Y Chebloune*3
}

\author{
Address: ${ }^{1}$ University of Kansas Medical Ctr, Dpt. of Microbiol Mol Genet \& immunol, Kansas City, KA, USA, ${ }^{2}$ Emory University, Atlanta, Georgia, \\ Dpt. of Pathol Lab Medicine, Atlanta, GA, USA and ${ }^{3}$ Animal Health, INRA, Villeurbanne, France \\ * Corresponding author
}

from AIDS Vaccine 2009

Paris, France. 19-22 October 2009

Published: 22 October 2009

Retrovirology 2009, 6(Suppl 3):P283 doi:10.1 186/1742-4690-6-S3-P283

This abstract is available from: http://www.retrovirology.com/content/6/S3/P283

(c) 2009 Arrode-Brusés et al; licensee BioMed Central Ltd.

\section{Background}

DNA vaccine is one of the most promising strategies for development of an efficacious HIV vaccine. Current HIV DNA vaccines induce potent immune responses (IR) in rodents but their immunogenicity remains weak in human and non-human primates (NHP). On the other hand, the MRKAd-5 based HIV vaccine that induces strong HIV-specific T cell responses failed to control HIV infection in human clinical trial. Therefore, strategies that enhance the immunogenicity of HIV DNA vaccines together with a strong commitment to better characterize the vaccine-induced IR are needed. Such comprehensive studies are critical in defining correlates of immune protection.

\section{Methods}

Accordingly, we used a single high dose of our $\triangle 4$ SHIVKU2 DNA vaccine to immunize 5 Rhesus monkeys and performed a longitudinal analysis of induced IR using multiparametric flow cytometry based assays.

\section{Results}

All 5 macaques developed long-lasting, potent $\mathrm{T}$ cell immune responses in their peripheral blood cells (PBMC). Interestingly, a peak of primary response was observed during the 4 weeks post-immunization. Then the response underwent a contraction phase and later reemerged in absence of any boost. The great majority of vaccine-specific CD4+ and CD8+ T cells lacked IFN- $\gamma$ pro- duction but showed high Ag-specific proliferation capacity. Proliferative CD8+ $\mathrm{T}$ cells expressed the lytic Granzyme B. Contrasting to these data no antibody response specific to HIV antigens was detected. This vaccination strategy was not associated with any detectable proviral integration in the PBMC of immunized animals. Furthermore no autoantibody to DNA was detected in sera of these animals.

\section{Conclusion}

Altogether, our comprehensive analysis demonstrated for the first time the capacity of a single high dose of HIV DNA vaccine alone to induce potent, long-lasting and polyfunctional T cell responses in NHP. 